(C2009 IEEE. Personal use of this material is permitted. However, permission to reprint/republish this material for advertising or promotional purposes or for creating new collective works for resale or redistribution to servers or lists, or to reuse any copyrighted component of this work in other works must be obtained from the IEEE. 


\section{Controlled and Conditioned Invariance with Stability for Two-Dimensional Systems}

\author{
Lorenzo Ntogramatzidis
}

\author{
Michael Cantoni
}

Ran Yang

\begin{abstract}
This paper collects, in a unified way, some recent results on a geometric approach to two-dimensional (2-D) system analysis and synthesis. The concepts of controlled and conditioned invariant subspaces, stabilisability and detectability subspaces, and output-nulling and input-containing subspaces, which prove useful in solving various 2-D filtering and decoupling problems, are developed for the Fornasini-Marchesini model in a general form.
\end{abstract}

\section{INTRODUCTION}

Over the last thirty years, a stream of literature has emerged regarding the modelling and analysis of twodimensional (2-D) systems, which operate between signals defined over a 2-D independent variable (e.g. space and time). This includes the well-known Roesser [16] and Fornasini-Marchesini (FM) [6], [7] models; these are inherently related as shown in [8], for example. Here, we consider the FM model in the generalised form of Kurek [12]:

$$
\begin{aligned}
x_{i+1, j+1}= & A_{0} x_{i, j}+A_{1} x_{i+1, j}+A_{2} x_{i, j+1} \\
& +B_{0} u_{i, j}+B_{1} u_{i+1, j}+B_{2} u_{i, j+1}, \\
y_{i, j}= & C x_{i, j}+D u_{i, j},
\end{aligned}
$$

where $x_{i, j} \in \mathbb{R}^{n}, u_{i, j} \in \mathbb{R}^{m}$ and $y_{i, j} \in \mathbb{R}^{p}$ are the local state, input and output at the signal index $(i, j)$, respectively.

The notions of controlled and conditioned invariance, first developed for 1-D systems by Basile and Marro in [1], are central to the so-called geometric approach to linear control system analysis and synthesis. Early results obtained in the development of a counterpart for 2-D systems can be found in [4], [11]. In particular, a definition of controlled invariance was first proposed in [4] for FM models. The definition, even though less powerful than its 1-D counterpart, enjoys feedback properties that are useful within the context of synthesis problems, including 2-D disturbance decoupling. Until recently [14], results pertaining to the computation of stabilising (local-)state feedback matrices were not available, which has limited application of these techniques, particularly from the perspective of numerical implementation.

This work was supported in part by the Australian Research Council (Discovery Project DP0986577), the National Natural Science Foundation of China (Grant No. 60504022) and the Natural Science Foundation of Guangdong (Grant No. 05003343).

L. Ntogramatzidis is with the Department of Mathematics and Statistics, Curtin University of Technology, Perth WA 6845, Australia. L.Ntogramatzidis@curtin.edu.au

M. Cantoni is with the Department of Electrical and Electronic Engineering, The University of Melbourne, Parkville VIC 3010, Australia. cantoni@unimelb.edu.au

R. Yang is with the School of Information Science and Technology, Sun Yat-Sen University, Guangzhou 510275, P.R. China. yangran@mail.sysu.edu.cn
In this paper, we collate recent work on controlled and conditioned invariance, with stability requirements. In particular, the following are provided:

(i) definitions for controlled and conditioned invariant subspaces for the 2-D model (1);

(ii) results regarding the basic properties of these subspaces and their characterisation in terms of local statefeedback and output injection, respectively;

(iii) corresponding definitions and analysis of stabilisability and detectability subpaces for the 2-D model (1);

(iv) LMI based analysis leading to sufficient conditions for the computation of stabilising local-state feedback and output injection matrices;

(v) corresponding definitions and analysis of output-nulling and input-containing subspaces for the 2-D model (1).

To achieve a unified development, we extend slightly the controlled invariance results of [14] to accommodate $A_{0}$ and $B_{0}$ in the model (1) and we provide new definitions of conditioned invariance compared to our initial work on this [15], where stability was not considered and a less general quotient observer structure is employed.

We denote the origin of $\mathbb{R}^{n}$ by $\mathbf{0}_{n}$. The image, kernel, transpose and Moore-Penrose inverse of a matrix $M$ are denoted $\operatorname{im} M, \operatorname{ker} M, M^{\top}$ and $M^{\dagger}$, respectively. The $n \times m$ zero matrix is denoted by $0_{n \times m}$. We defined $M_{D} \triangleq \operatorname{diag}(M, M, M)$, and, accordingly, given a subspace $\mathscr{J} \subseteq \mathbb{R}^{n}$, the symbol $\mathscr{J}_{D}$ denotes the subspace $\mathscr{J} \times \mathscr{J} \times \mathscr{J}$ of $\mathbb{R}^{3 n}$, where $\times$ is the Cartesian product. Given the vector $\xi \in \mathbb{R}^{n}$, the symbol $\xi / \mathscr{J}$ denotes the canonical projection of $\xi$ on the quotient space $\mathbb{R}^{n} / \mathscr{J}$. Finally, given a triple of matrices $\left(M_{0}, M_{1}, M_{2}\right)$, we define $M_{H} \triangleq\left[\begin{array}{lll}M_{0} & M_{1} & M_{2}\end{array}\right]$ and $M_{V} \triangleq$ $\left[\begin{array}{lll}M_{0}^{\top} & M_{1}^{\top} & M_{2}^{\top}\end{array}\right]^{\top}$.

\section{INVARIANT SUBSPACES FOR FM MODElS}

We begin by considering the autonomous FM model

$$
x_{i+1, j+1}=A_{0} x_{i, j}+A_{1} x_{i+1, j}+A_{2} x_{i, j+1} .
$$

As boundary conditions for (2) we use $x_{i, j}=b_{i, j} \in \mathbb{R}^{n}$ for all $(i, j) \in \mathfrak{B}$ and some constants $b_{i, j} \in \mathbb{R}^{n}$, where $\mathfrak{B} \triangleq(\mathbb{N} \times$ $\{0\}) \cup(\{0\} \times \mathbb{N}) .^{1}$

A subspace $\mathscr{J}$ of $\mathbb{R}^{n}$ is said to be $\left(A_{0}, A_{1}, A_{2}\right)$-invariant if $\mathscr{J}$ is $A_{i}$-invariant for $i \in\{0,1,2\}$ in the usual 1-D sense; i.e., $A_{i} x \in \mathscr{J}$ for all $x \in \mathscr{J}$ and $i \in\{0,1,2\}$. The following provides geometric and matrix conditions for invariance.

Lemma 1: The following are equivalent:

${ }^{1}$ Other choices of $\mathfrak{B}$, for which a unique solution of (2) exists, are possible; see [7]. The results in this paper can be adapted to these cases. 
1) $\mathscr{J}$ is $\left(A_{0}, A_{1}, A_{2}\right)$-invariant;

2) $A_{V} \mathscr{J} \subseteq \mathscr{J}_{D}$;

3) $A_{H} \mathscr{J}_{D} \subseteq \mathscr{J}$;

4) There exist $X_{0}, X_{1}, X_{2} \in \mathbb{R}^{r \times r}$ such that $A_{i} J=J X_{i}$, where $J \in \mathbb{R}^{n \times r}$ is a full column-rank matrix such that $\operatorname{im} J=$ $\mathscr{J}$, i.e., $A_{V} J=J_{D} X_{V}$;

5) There exist $L_{0}, L_{1}, L_{2} \in \mathbb{R}^{(n-r) \times(n-r)}$ such that $Q A_{i}=$ $L_{i} Q$ for $i \in\{0,1,2\}$, where $Q \in \mathbb{R}^{r \times n}$ is a full row-rank matrix such that $\operatorname{ker} Q=\mathscr{J}$, i.e., $Q A_{H}=L_{H} Q_{D}$.

Proof: 1) $\Longrightarrow$ 2) This follows directly by definition.

2) $\Longrightarrow$ 3) For $\xi_{i} \in \mathscr{J}, i \in\{0,1,2\}$, it follows that $A_{i} \xi_{i} \in \mathscr{J}$, and hence, $A_{0} \xi_{0}+A_{1} \xi_{1}+A_{2} \xi_{2}=A_{H}\left[\begin{array}{lll}\xi_{0}^{\top} & \xi_{1}^{\top} & \xi_{2}^{\top}\end{array}\right]^{\top} \in \mathscr{J}$. 3) $\Longrightarrow$ 1) Suppose there exist an $i \in\{0,1,2\}$ and an $\xi \in$ $\mathscr{J}$ such that $A_{i} \xi \notin \mathscr{J}$; i.e., $\mathscr{J}$ is not $\left(A_{0}, A_{1}, A_{2}\right)$-invariant. Then $A_{H}\left[\begin{array}{lll}\xi^{\top} & 0 & 0\end{array}\right]^{\top} \notin \mathscr{J}$, which contradicts 3).

2) $\Leftrightarrow$ 4) Note that 2) is equivalent to $\operatorname{im} A_{V} J \subseteq \operatorname{im} J_{D}$, by which the result holds, since for any matrices $Y \in \mathbb{R}^{p \times m}$ and $Z \in \mathbb{R}^{p \times q}, \operatorname{im} Y \subseteq \operatorname{im} Z$ if, and only if, there exist an $X \in$ $\mathbb{R}^{q \times m}$ such that $Y=Z X$; when $Z$ has full column-rank then $X=\left(Z^{\top} Z\right)^{-1} Z^{\top} Y=Z^{\dagger} Y$ is the unique solution.

3) $\Leftrightarrow$ 5) Note that 3) is equivalent to $\operatorname{ker} Q_{D} \subseteq \operatorname{ker} Q A_{H}$, by which the result holds, since for any matrices $M \in \mathbb{R}^{p \times m}$ and $N \in \mathbb{R}^{q \times p}$, $\operatorname{ker} M \subseteq \operatorname{ker} N$ if, and only if, there exist an $L \in \mathbb{R}^{q \times m}$ such that $N=L M$; when $M$ has full row-rank then $L=N M^{\top}\left(M M^{\top}\right)^{-1}=N M^{\dagger}$ is the unique solution.

The following theorem is the 2-D counterpart of a wellknown result (see [1]) concerning the decomposition of a 1-D system matrix with respect to an invariant subspace.

Theorem 1: The following are equivalent:

1) There exists an $r$-dimensional subspace $\mathscr{J} \subseteq \mathbb{R}^{n}$ that is $\left(A_{0}, A_{1}, A_{2}\right)$-invariant;

2) There exists a similarity transformation $T \in \mathbb{R}^{n \times n}$ such that for each $i \in\{0,1,2\}$

$$
\hat{A}_{i} \triangleq T^{-1} A_{i} T=\left[\begin{array}{cc}
\hat{A}_{i}^{11} & \hat{A}_{i}^{12} \\
0_{(n-r) \times r} & \hat{A}_{i}^{22}
\end{array}\right] .
$$

Proof: 1) $\Longrightarrow$ 2) Let $J \in \mathbb{R}^{n \times r}$ be a basis matrix for $\mathscr{J}$. Then, by Lemma 1 , three matrices $X_{0}, X_{1}, X_{2} \in \mathbb{R}^{r \times r}$ exist such that $A_{i} J=J X_{i}$ for all $i \in\{0,1,2\}$. Since $J$ is of full column-rank, a non-singular matrix $T \in \mathbb{R}^{n \times n}$ exists such that $T^{-1} J=\left[\begin{array}{c}I_{r} \\ 0_{(n-r) \times r}\end{array}\right]$. As such, with $\hat{A}_{i}=T^{-1} A_{i} T=\left[\begin{array}{cc}\hat{A}_{i}^{11} & \hat{A}_{i}^{12} \\ \hat{A}_{i}^{21} & \hat{A}_{i}^{22}\end{array}\right]$ for $i \in\{0,1,2\}$, it follows that

$$
\begin{gathered}
{\left[\begin{array}{c}
\hat{A}_{i}^{11} \\
\hat{A}_{i}^{21}
\end{array}\right]=\left[\begin{array}{cc}
\hat{A}_{i}^{11} & \hat{A}_{i}^{12} \\
\hat{A}_{i}^{21} & \hat{A}_{i}^{22}
\end{array}\right]\left[\begin{array}{c}
I_{r} \\
0_{(n-r) \times r}
\end{array}\right]=\hat{A}_{i} T^{-1} J} \\
\quad=T^{-1} A_{i} J=T^{-1} J X_{i}=\left[\begin{array}{c}
X_{i} \\
0_{(n-r) \times r}
\end{array}\right]
\end{gathered}
$$

for $i \in\{0,1,2\}$. That is, $\hat{A}_{i}^{21}=0$ as required.

2) $\Longrightarrow 1$ ) Let $T$ be such that (3) holds. Then,

$$
\hat{A}_{i}\left[\begin{array}{c}
I_{r} \\
0_{(n-r) \times r}
\end{array}\right]=\left[\begin{array}{c}
X_{i} \\
0_{(n-r) \times r}
\end{array}\right]
$$

holds for $X_{i}=\hat{A}_{i}^{11}$. Pre-multiplying (4) by $T$ yields

$$
A_{i} T\left[\begin{array}{c}
I_{r} \\
0_{(n-r) \times r}
\end{array}\right]=T\left[\begin{array}{c}
I_{r} \\
0_{(n-r) \times r}
\end{array}\right] X_{i} .
$$

As such, $T\left[\begin{array}{c}I_{r} \\ 0_{(n-r) \times r}\end{array}\right]$ is an $r$-dimensional $\left(A_{0}, A_{1}, A_{2}\right)$ invariant subspace, by Lemma 1.

\section{A. Invariant Subspaces and Local-State Trajectories}

Lemma 2: Consider an $\left(A_{0}, A_{1}, A_{2}\right)$-invariant subspace $\mathscr{J}$. A boundary condition $x_{i, j}=b_{i, j} \in \mathscr{J}$, for $(i, j) \in \mathfrak{B}$, gives rise to $x_{i, j} \in \mathscr{J}$ for all $i, j \geq 0$.

Proof: In the set of coordinates corresponding to any similarity transformation $T=\left[\begin{array}{ll}T_{1} & T_{2}\end{array}\right]$, with $\operatorname{im} T_{1}=\mathscr{J}$, and partitioning local state comformably with $T$, as $\left[\begin{array}{l}x_{i, j}^{\prime} \\ x_{i, j}^{\prime \prime}\end{array}\right]=$ $T^{-1} x_{i, j}$, it follows by Theorem 1 that

$$
\begin{aligned}
{\left[\begin{array}{c}
x_{i+1, j+1}^{\prime} \\
x_{i+1, j+1}^{\prime \prime}
\end{array}\right]=} & {\left[\begin{array}{cc}
\hat{A}_{0}^{11} & \hat{A}_{0}^{12} \\
0 & \hat{A}_{0}^{22}
\end{array}\right]\left[\begin{array}{l}
x_{i, j}^{\prime} \\
x_{i, j}^{\prime \prime}
\end{array}\right]+\left[\begin{array}{cc}
\hat{A}_{1}^{11} & \hat{A}_{1}^{12} \\
0 & \hat{A}_{1}^{22}
\end{array}\right]\left[\begin{array}{c}
x_{i+1, j}^{\prime} \\
x_{i+1, j}^{\prime \prime}
\end{array}\right] } \\
& +\left[\begin{array}{cc}
\hat{A}_{2}^{11} & \hat{A}_{2}^{12} \\
0 & \hat{A}_{2}^{22}
\end{array}\right]\left[\begin{array}{c}
x_{i, j+1}^{\prime} \\
x_{i, j+1}^{\prime \prime}
\end{array}\right]
\end{aligned}
$$

Note that any boundary condition $x_{i, j}=b_{i, j} \in \mathscr{J}$ is such that $x_{i, j}^{\prime \prime}=0$ for $(i, j) \in \mathfrak{B}$. Moreover, by $(5), x_{i, j}^{\prime \prime}=0$ for all $i, j \geq 0$. Hence, $x_{i, j} \in \mathscr{J}$ for all $i, j \geq 0$.

In the basis corresponding to $T$ in Lemma 2, the component $x_{i, j}^{\prime}$ is the projection of the local state $x_{i, j}$ onto the invariant subspace $\mathscr{J}$, while $x_{i, j}^{\prime \prime}$ is the canonical projection on to the quotient space $\mathbb{R}^{n} / \mathscr{J}$.

\section{B. Internal and External Stability of Invariant Subspaces}

A necessary and sufficient condition for asymptotic stability of (2) - often said asymptotic stability of the triple $\left(A_{0}, A_{1}, A_{2}\right)-$ is that $\forall\left(z_{1}, z_{2}\right) \in \mathfrak{P}$

$$
\operatorname{det}\left(I_{n}-A_{0} z_{1} z_{2}-A_{1} z_{2}-A_{2} z_{1}\right) \neq 0,
$$

where $\mathfrak{P} \triangleq\left\{\left(\zeta_{1}, \zeta_{2}\right) \in \mathbb{C} \times \mathbb{C}|| \zeta_{1} \mid<1\right.$ and $\left.\left|\zeta_{2}\right|<1\right\}$; this is equivalent to $x_{i, j} \rightarrow 0$ as $i+j \rightarrow \infty$. Various, more computationally tractable, sufficient stability conditions have been proposed over the last two decades, in terms of Lyapunov equations and/or spectral radius conditions of certain matrices, see e.g. [9], [10], [3]. In the very recent literature, new necessary and sufficient criteria have appeared for asymptotic stability in terms of conditions that can be checked in finite terms, see [17], [5]. For the sake of argument and clarity, however, the following simple sufficient condition for asymptotic stability, expressed in terms of an linear matrix inequality (LMI), will be used herein:

Lemma 3: ([10]) The triple $\left(A_{0}, A_{1}, A_{2}\right)$ is asymptotically stable if three symmetric positive definite matrices $P_{0}, P_{1}$ and $P_{2}$ exist such that:

$$
\operatorname{diag}\left(P_{0}, P_{1}, P_{2}\right)-A_{H}^{\top}\left(P_{0}+P_{1}+P_{2}\right) A_{H}>0 .
$$

We now show that stability of (2) can be "split" into two parts with respect to an invariant subspace $\mathscr{J} \subseteq \mathbb{R}^{n \times n}$. Expressing (2) in the set of coordinates corresponding to the similarity transformation $T$ in Theorem 1 ,

$$
\begin{aligned}
\operatorname{det}\left(I_{n}-\right. & \left.\hat{A}_{0} z_{1} z_{2}-\hat{A}_{1} z_{2}-\hat{A}_{2} z_{1}\right) \\
= & \operatorname{det}\left(I-\hat{A}_{0}^{11} z_{1} z_{2}-\hat{A}_{1}^{11} z_{2}-\hat{A}_{2}^{11} z_{1}\right) \\
& \cdot \operatorname{det}\left(I-\hat{A}_{0}^{22} z_{1} z_{2}-\hat{A}_{1}^{22} z_{2}-\hat{A}_{2}^{22} z_{1}\right) .
\end{aligned}
$$


It follows that (2) is asymptotically stable if and only if $\left(\hat{A}_{0}^{11}, \hat{A}_{1}^{11}, \hat{A}_{2}^{11}\right)$ and $\left(\hat{A}_{0}^{22}, \hat{A}_{1}^{22}, \hat{A}_{2}^{22}\right)$ are asymptotically stable.

Definition 1: The $\left(A_{0}, A_{1}, A_{2}\right)$-invariant subspace $\mathscr{J}$ is

- internally stable if the corresponding triple $\left(\hat{A}_{0}^{11}, \hat{A}_{1}^{11}, \hat{A}_{2}^{11}\right)$ is asymptotically stable.

- externally stable if the corresponding triple $\left(\hat{A}_{0}^{22}, \hat{A}_{1}^{22}, \hat{A}_{2}^{22}\right)$ is asymptotically stable.

Hence, (2) is asymptotically stable if and only if any invariant subspace is both internally and externally stable.

Corollary 1: Given the $r$-dimensional subspace $\mathscr{J}$ of $\mathbb{R}^{n}$, let $J \in \mathbb{R}^{n \times r}$ be a full column-rank matrix such that im $J=$ $\mathscr{J}$ and $Q \in \mathbb{R}^{(n-r) \times n}$ be a full row-rank matrix such that $\operatorname{ker} Q=\mathscr{J}$. Then

1) $\mathscr{J}$ is an internally stable $\left(A_{0}, A_{1}, A_{2}\right)$-invariant subspace if, and only if, an asymptotically stable triple $\left(X_{0}, X_{1}, X_{2}\right)$ exists such that $A_{i} J=J X_{i}$ for all $i \in$ $\{0,1,2\}$

2) $\mathscr{J}$ is an externally stable $\left(A_{0}, A_{1}, A_{2}\right)$-invariant subspace if, and only if, an asymptotically stable triple $\left(L_{0}, L_{1}, L_{2}\right)$ exists such that $Q A_{i}=L_{i} Q$ for all $i \in$ $\{0,1,2\}$.

Proof: Consider 1). With respect to a basis of $\mathbb{R}^{n}$ adapted to $\mathscr{J}$, the identity $A_{i} J=J X_{i}$ can be written as

$$
\left[\begin{array}{cc}
A_{i}^{11} & A_{i}^{12} \\
0_{(n-r) \times r} & A_{i}^{22}
\end{array}\right]\left[\begin{array}{c}
I_{r} \\
0_{(n-r) \times r}
\end{array}\right]=\left[\begin{array}{c}
I_{r} \\
0_{(n-r) \times r}
\end{array}\right] X_{i}
$$

for $i \in\{0,1,2\}$, so that $A_{i}^{11}=X_{i}$. Now consider 2). With respect to a basis of $\mathbb{R}^{n}$ adapted to $\mathscr{J}, Q=$ $\left[0_{(n-r) \times r} I_{(n-r) \times(n-r)}\right]$ is a full row-rank matrix such that $\operatorname{ker} Q=\mathscr{J}$. Writing the identities $Q A_{i}=L_{i} Q$ for all $i \in$ $\{0,1,2\}$ with respect to this basis yields

$$
\left[\begin{array}{ll}
0_{(n-r) \times r} & I_{(n-r)}
\end{array}\right]\left[\begin{array}{rr}
A_{i}^{11} & A_{i}^{12} \\
0 & A_{i}^{22}
\end{array}\right]=L_{i}\left[\begin{array}{ll}
0_{(n-r) \times r} & I_{(n-r)}
\end{array}\right],
$$

leading to $L_{i}=A_{i}^{22}$ for all $i \in\{0,1,2\}$.

\section{CONTROlled INVARIANT SUbSPACES}

Consider the non-autonomous FM model (1) Again, the boundary conditions associated with this model corresponding to specifying the local state over $\mathfrak{B}$.

Definition 2: ([4]) The subspace $\mathscr{V} \subseteq \mathbb{R}^{n}$ is controlled invariant for (1) if

$$
A_{V} \mathscr{V} \subseteq \mathscr{V}_{D}+\operatorname{im} B_{V}
$$

A direct consequence of this definition is that the subspaces $\mathbf{0}_{n}$ and $\mathbb{R}^{n}$ are controlled invariant subspaces for (1). It is easy to see that the set of controlled invariant subspaces is closed under subspace addition. Moreover, if $\mathscr{V}$ is controlled invariant then it is both $\left(A_{1}, B_{1}\right)$ and $\left(A_{2}, B_{2}\right)$ controlled invariant in the usual 1-D sense [1]. The converse, however, is not true in general, as observed in [11]. A controlled invariant subspace $\mathscr{V}$ implies the existence of a set of inputs $\left\{u_{i, j} \mid i, j \geq 0\right\}$ for which the corresponding local state solution of (1) lies in $\mathscr{V}$, for all $i, j>0$ and any $\mathscr{V}$-valued boundary condition. While in the $1-\mathrm{D}$ case the converse is true as well, with this definition the subspace of minimal dimension which contains a given sequence satisfying (1) is not necessarily controlled invariant. Nonetheless, this definition enjoys good feedback properties, as shown for the first time in [4], and briefly recalled in Lemma 4.

Lemma 4: Let $\mathscr{V}$ be an $r$-dimensional subspace and let $V \in \mathbb{R}^{n \times r}$ be a basis matrix for $\mathscr{V}$. The following are equivalent:

1) The subspace $\mathscr{V}$ is controlled invariant for (1);

2) Matrices $X \in \mathbb{R}^{3 r \times r}$ and $\Omega \in \mathbb{R}^{m \times r}$ exist such that

$$
A_{V} V=V_{D} X+B_{V} \Omega
$$

3) A matrix $F \in \mathbb{R}^{m \times n}$ exists such that with $A_{i}^{F} \triangleq A_{i}+B_{i} F$, $\mathscr{V}$ is $\left(A_{0}^{F}, A_{1}^{F}, A_{2}^{F}\right)$-invariant, i.e.,

$$
A_{V}^{F} \mathscr{V} \subseteq \mathscr{V}_{D}
$$

4) Matrices $F \in \mathbb{R}^{m \times n}$ and $X \in \mathbb{R}^{3 r \times r}$ exist such that

$$
A_{V}^{F} V=V_{D} X \text {. }
$$

Proof: The implication 1) $\Longrightarrow$ 2) follows from Definition 2 on noting that (10) is simply a matrix representation of the subspace inclusion (9). To prove 2) $\Longrightarrow 3$ ) it suffices to take $F=-\Omega\left(V^{\top} V\right)^{-1} V^{\top}$. It follows that $\Omega=-F V$, that can be used in (10) to get (11). The result 3) $\Longrightarrow 4$ ) follows directly from the fact that (12) is a matrix representation of the inclusion (11). Finally, the implication 4) $\Longrightarrow$ 1) follows by re-writing (12) as

$$
A_{V} V=V_{D} X-B_{V} F V
$$

by which (9) holds, completing the proof.

Remark 1: For a controlled invariant subspace $\mathscr{V}=\operatorname{im} V$, with $\operatorname{ker} V=\{0\}$, pairs of matrices $X$ and $\Omega$ that satisfy the linear equation (10) can be parameterised by

$$
\left[\begin{array}{l}
X \\
\Omega
\end{array}\right]=W^{\dagger} A_{V} V+H K
$$

where $W \triangleq\left[\begin{array}{ll}V_{D} & B_{V}\end{array}\right], H$ is a basis matrix for $\operatorname{ker} W$ and $K$ is an arbitrary matrix of suitable size.

Let $F$ be such that (11) holds true. Applying a static localstate feedback $u_{i, j}=F x_{i, j}$ in (1) we find that

$$
x_{i+1, j+1}=A_{0}^{F} x_{i, j}+A_{1}^{F} x_{i+1, j}+A_{2}^{F} x_{i, j+1} .
$$

Moreover, under such control action and given a $\mathscr{V}$-valued boundary condition, i.e., such that $x_{i, j} \in \mathscr{V}$ for all $(i, j) \in \mathfrak{B}$, it follows as in the autonomous case discussed above, that $x_{i, j} \in \mathscr{V}$ for all $i, j \in \mathbb{N}$. The set of matrices $F$ such that (11) holds is denoted by $\mathfrak{F}\left(\mathscr{V}, \Sigma_{0}\right)$; when $F \in \mathfrak{F}\left(\mathscr{V}, \Sigma_{0}\right)$ it is said to be a friend of the controlled invariant subspace $\mathscr{V}$. As in the 1-D case, and since $\mathscr{V}$ is $\left(A_{0}^{F}, A_{1}^{F}, A_{2}^{F}\right)$-invariant for all $F \in \mathfrak{F}(\mathscr{V})$, the definitions for internal and external stability can be used to define notions of internal and external stabilisability with respect to a controlled invariant subspace.

Definition 3: The controlled invariant subspace $\mathscr{V}$ is said to be internally (resp. externally) stabilisable if there exists an $F \in \mathfrak{F}\left(\mathscr{V}, \Sigma_{0}\right)$ such that $\mathscr{V}$ is an internally (resp. externally) stable $\left(A_{0}^{F}, A_{1}^{F}, A_{2}^{F}\right)$-invariant subspace. An internally stabilisable controlled invariant subspace is sometimes called a stabilisability subspace. 
To see how to choose a friend $F$ of a controlled invariant subspace $\mathscr{V}$ to achieve internally (resp. externally) stability, a more explicit characterisation of the set $\mathfrak{F}\left(\mathscr{V}, \Sigma_{0}\right)$ is required.

Lemma 5: Let $\mathscr{V}$ be an $r$-dimensional controlled invariant subspace and let $V \in \mathbb{R}^{n \times r}$ be a basis matrix for $\mathscr{V}$. Each matrix $F \in \mathfrak{F}\left(\mathscr{V}, \Sigma_{0}\right)$ is a solution of the linear equation $\Omega=$ $-F V$, where $\Omega \in \mathbb{R}^{m \times r}$ is a solution of (10) for some $X \in$ $\mathbb{R}^{3 r \times r}$. In particular,

$$
\mathfrak{F}\left(\mathscr{V}, \Sigma_{0}\right)=\left\{F=-\Omega\left(V^{\top} V\right)^{-1} V^{\top}+\tilde{F} \mid\right.
$$

$\Omega$ satisfies (10) for some $X$ and $\tilde{F} V=0\}$.

Proof: The statement follows on noting that any $F \in$ $\mathfrak{F}\left(\mathscr{V}, \Sigma_{0}\right)$ satisfies (12) for some $X \in \mathbb{R}^{3 r \times r}$. Hence, (12) can be written as (13). It follows that (10) is satisfied with this $X$ and $\Omega=-F V$. To complete the proof, note that since $V$ is full column-rank, all solutions of $\Omega=-F V$ can be written as

$$
F=F_{\Omega}+\tilde{F},
$$

where $F_{\Omega}=-\Omega\left(V^{\top} V\right)^{-1} V^{\top}$ and $\tilde{F}$ is any matrix of suitable size such that $\tilde{F} V=0$.

Since all $F \in \mathfrak{F}\left(\mathscr{V}, \Sigma_{0}\right)$ are such that $\mathscr{V}$ is $\left(A_{0}^{F}, A_{1}^{F}, A_{2}^{F}\right)$ invariant, the similarity transformation $T=\left[\begin{array}{ll}T_{1} & T_{2}\end{array}\right]$, with $T_{1}$ set to be a basis matrix for $\mathscr{V}$, is such that

$$
T^{-1}\left(A_{i}+B_{i} F\right) T=\left[\begin{array}{cc}
\Delta_{i}^{11}(\Omega, \tilde{F}) & \Delta_{i}^{12}(\Omega, \tilde{F}) \\
0 & \Delta_{i}^{22}(\Omega, \tilde{F})
\end{array}\right]
$$

for $i \in\{0,1,2\}$. Equation (18) emphasises that for different values of $\Omega$ and $\tilde{F}$ satisfying the conditions in (16), we obtain different matrices $\Delta_{i}^{\star}(\Omega, \tilde{F})$. Importantly, it is shown in Lemma 6 below that the matrices $\Delta_{1}^{11}(\Omega, \tilde{F})$ and $\Delta_{2}^{11}(\Omega, \tilde{F})$ do not depend on $\tilde{F}$, and similarly, the matrices $\Delta_{1}^{22}(\Omega, \tilde{F})$ and $\Delta_{2}^{22}(\Omega, \tilde{F})$ do not depend on $\Omega$. In this way, the two matrices $\Omega$ and $\tilde{F}$ can be chosen independently to build a friend of $\mathscr{V}$, so that the former does not affect $\left(\Delta_{1}^{22}, \Delta_{2}^{22}\right)$ and the latter does not affect $\left(\Delta_{1}^{11}, \Delta_{2}^{11}\right)$. In other words, when $\mathscr{V}$ is internally stabilisable, $\Omega$ can be chosen first so that $F_{\Omega}$ stabilises $\left(\Delta_{1}^{11}, \Delta_{2}^{11}\right)$, and then $\tilde{F}$ can be chosen to stabilise $\left(\Delta_{1}^{22}, \Delta_{2}^{22}\right)$, if $\mathscr{V}$ is also externally stabilisable, without affecting the internal stabilisation achieved with $F_{\Omega}$. These two independent stabilisation procedures are examined in the remainder of this section.

Lemma 6: The matrices $\Delta_{i}^{11}(\Omega, \tilde{F})$ in (18) do not depend on $\tilde{F}$. The matrices $\Delta_{i}^{22}(\Omega, \tilde{F})$ in (18) do not depend on $\Omega$. Proof: First, we prove that the matrices $\Delta_{i}^{11}(\Omega, \tilde{F})$ in (18) do not depend on $\tilde{F}$. Let $F_{k}=F_{\Omega}+\tilde{F}_{k}$ for $k=1,2$, where $\tilde{F}_{1}$ and $\tilde{F}_{2}$ are such that $\tilde{F}_{1} V=0$ and $\tilde{F}_{2} V=0$, and $F_{\Omega}=$ $-\Omega\left(V^{\top} V\right)^{-1} V^{\top}$, where $\Omega$ is such that (10) holds for some $X$. Then, (18) can be written as

$$
T^{-1}\left(A_{i}+B_{i} F_{k}\right) T=\left[\begin{array}{cc}
\Delta_{i}^{11}\left(\Omega, \tilde{F}_{k}\right) & \Delta_{i}^{12}\left(\Omega, \tilde{F}_{k}\right) \\
0 & \Delta_{i}^{22}\left(\Omega, \tilde{F}_{k}\right)
\end{array}\right] .
$$

Our aim is to show that $\Delta_{i}^{11}\left(\Omega, \tilde{F}_{1}\right)=\Delta_{i}^{11}\left(\Omega, \tilde{F}_{2}\right)$ for $i=1,2$. From (19) we find

$$
\begin{aligned}
& {\left[\begin{array}{cc}
\Delta_{i}^{11}\left(\Omega, \tilde{F}_{1}\right)-\Delta_{i}^{11}\left(\Omega, \tilde{F}_{2}\right) & \Delta_{i}^{12}\left(\Omega, \tilde{F}_{1}\right)-\Delta_{i}^{12}\left(\Omega, \tilde{F}_{2}\right) \\
0 & \Delta_{i}^{22}\left(\Omega, \tilde{F}_{1}\right)-\Delta_{i}^{22}\left(\Omega, \tilde{F}_{2}\right)
\end{array}\right]} \\
& =T^{-1}\left(A_{i}+B_{i} F_{\Omega}+B_{i} \tilde{F}_{1}\right) T-T^{-1}\left(A_{i}+B_{i} F_{\Omega}+B_{i} \tilde{F}_{2}\right) T \\
& =T^{-1} B_{i}\left(\tilde{F}_{1}-\tilde{F}_{2}\right)\left[\begin{array}{ll}
T_{1} & T_{2}
\end{array}\right]=\left[\begin{array}{ll}
0 & T^{-1} B_{i}\left(\tilde{F}_{1}-\tilde{F}_{2}\right) T_{2}
\end{array}\right],
\end{aligned}
$$

since $\tilde{F}_{1} T_{1}=\tilde{F}_{2} T_{1}=0$. Thus, $\Delta_{i}^{11}\left(\Omega, \tilde{F}_{1}\right)-\Delta_{i}^{11}\left(\Omega, \tilde{F}_{2}\right)=0$.

Now we show that the matrices $\Delta_{i}^{22}(\Omega, \tilde{F})$ in (18) do not depend on $\Omega$. To this end, let $\Omega_{1}$ and $\Omega_{2}$ be such that (10) holds for some $X_{1}$ and $X_{2}$, respectively. By difference,

$$
V_{D}\left(X_{1}-X_{2}\right)+B_{V}\left(\Omega_{1}-\Omega_{2}\right)=0 .
$$

With $F_{k}=-\Omega_{k}\left(V^{\top} V\right)^{-1} V^{\top}+\tilde{F}$, for $k=1,2$, where $\tilde{F}$ is any matrix such that $\tilde{F} V=0$, it follows that (18) can be written as

$$
T^{-1}\left(A_{i}+B_{i} F_{k}\right) T=\left[\begin{array}{cc}
\Delta_{i, 11}\left(\Omega_{k}, \tilde{F}\right) & \Delta_{i, 12}\left(\Omega_{k}, \tilde{F}\right) \\
0 & \Delta_{i, 22}\left(\Omega_{k}, \tilde{F}\right)
\end{array}\right] .
$$

For the sake of conciseness, let $L_{i, \star} \triangleq \Delta_{i, \star}\left(\Omega_{1}, \tilde{F}\right)-$ $\Delta_{i, \star}\left(\Omega_{2}, \tilde{F}\right)$. Subtracting (21), with $k=2$, from (21), with $k=1$, gives

$B_{i}\left(\Omega_{2}-\Omega_{1}\right)\left(V^{\top} V\right)^{-1} V^{\top}\left[\begin{array}{ll}T_{1} & T_{2}\end{array}\right]=\left[\begin{array}{cc}T_{1} & T_{2}\end{array}\right]\left[\begin{array}{cc}L_{i, 11} & L_{i, 12} \\ 0 & L_{i, 22}\end{array}\right]$

which in particular, yields $B_{i}\left(\Omega_{2}-\Omega_{1}\right)\left(V^{\top} V\right)^{-1} V^{\top} T_{2}=$ $T_{1} L_{i, 12}+T_{2} L_{i, 22}$. Since no generality is lost by assuming $T_{1}=V$, we find that

$$
B_{V}\left(\Omega_{2}-\Omega_{1}\right)\left(V^{\top} V\right)^{-1} V^{\top} T_{2}=\left[\begin{array}{c}
V L_{1,12}+T_{2} L_{1,22} \\
V L_{2,12}+T_{2} L_{2,22}
\end{array}\right] .
$$

Then using (20) to obtain

$$
V_{D}\left(X_{1}-X_{2}\right)\left(V^{\top} V\right)^{-1} V^{\top} T_{2}=V_{D}\left[\begin{array}{l}
L_{1,12} \\
L_{2,12}
\end{array}\right]+\left[\begin{array}{cc}
T_{2} & 0 \\
0 & T_{2}
\end{array}\right]\left[\begin{array}{l}
L_{1,22} \\
L_{2,22}
\end{array}\right]
$$

it follows that $\left[\begin{array}{cc}T_{2} & 0 \\ 0 & T_{2}\end{array}\right]\left[\begin{array}{l}L_{1,22} \\ L_{2,22}\end{array}\right]=0$, since $V$ and $T_{2}$ have linearly independent columns. This in turns implies that $L_{1,22}=L_{2,22}=0$ since $T_{2}$ has linearly independent columns. This means $\Delta_{i, 22}\left(\Omega_{1}, \tilde{F}\right)=\Delta_{i, 22}\left(\Omega_{2}, \tilde{F}\right)$ for $i \in\{0,1,2\}$.

Finding an $F_{\Omega}$ to internally stabilise $\mathscr{V}$ is equivalent to finding an $F_{\Omega}$ for which a triple $\left(X_{0}, X_{1}, X_{2}\right)$ satisfying (12) is asymptotically stable. Since the only degree of freedom lies in the choice of $\Omega$, which is given by (14), we have the following.

- When the nullspace of $W \triangleq\left[V_{D} B_{V}\right]$ is zero, i.e., when

$$
\mathscr{V}_{D} \cap \operatorname{im} B_{V}=\mathbf{0}_{3 n}
$$

there is only one solution to (10), and this either achieves internal stabilisation or it does not.

- When $W$ has non-trivial kernel, we can write (14) as

$$
\left[\begin{array}{c}
X_{0} \\
X_{1} \\
X_{2} \\
\Omega
\end{array}\right]=\left[\begin{array}{l}
L_{0} \\
L_{1} \\
L_{2} \\
L_{3}
\end{array}\right]+\left[\begin{array}{l}
H_{0} \\
H_{1} \\
H_{2} \\
H_{3}
\end{array}\right] K,
$$

where $\quad\left[\begin{array}{cccc}L_{0}^{\top} & L_{1}^{\top} & L_{2}^{\top} & L_{3}^{\top}\end{array}\right]^{\top} \triangleq W^{\dagger} A_{V} V$, $\operatorname{im}\left[\begin{array}{llll}H_{0}^{\top} & H_{1}^{\top} & H_{2}^{\top} & H_{3}^{\top}\end{array}\right]^{\top}=\operatorname{ker} W$ and $K$ is an arbitrary matrix of suitable size. The problem of internal stabilization with respect to $\mathscr{V}$ then becomes one of finding a $K$ such that $\left(X_{0}, X_{1}, X_{2}\right)$ is asymptotically stable. If such a $K$ exists, we can exploit it in order to compute $\Omega$ from (23) along with the 
corresponding asymptotically stable triple $\left(X_{0}, X_{1}, X_{2}\right)$, to yield the required solution of (10). Moreover, with $F=-\Omega\left(V^{\top} V\right)^{-1} V^{\top}$, we find that (12) is also satisfied, which implies $F$ internally stabilises $\mathscr{V}$.

The following result provides a computationally tractable sufficient condition for the internal stabilisability of a controlled invariant subspace.

Theorem 2: The controlled invariant subspace $\mathscr{V}$ is internally stabilisable if there exist matrices $M=M^{\top}>0$, $N=N^{\top}>0, Z=Z^{\top}>0$ and $E$, of suitable dimensions, such that

$$
\left[\begin{array}{cccc}
-M & 0 & 0 & \tilde{Z}_{0}^{\top} \\
0 & -N & 0 & \tilde{Z}_{1}^{\top} \\
0 & 0 & -(Z-M-N) & \tilde{Z}_{2}^{\top} \\
\tilde{Z}_{0} & \tilde{Z}_{1} & \tilde{Z}_{2} & -Z
\end{array}\right]<0 .
$$

where $\tilde{Z}_{i} \triangleq L_{i} Z+H_{i} E$ and $L_{i}$ and $H_{i}$ are as defined in (23). Given a $(M, N, E)$ in the convex set defined by (24), $K=E Z^{-1}$ is such that the corresponding triple $\left(X_{0}, X_{1}, X_{2}\right)$ obtained from (23) is asymptotically stable.

Proof: The controlled invariant subspace $\mathscr{V}$ is internally stabilisable if, and only if, there exist symmetric positive definite matrices $P_{0}, P_{1}$ and $P_{2}$ such that the triple $\left(X_{0}, X_{1}, X_{2}\right)$ satisfies (7) in Lemma 3. Since $X_{i}=L_{i}+H_{i} K(i=0,1,2)$, this is equivalent to the existence of three symmetric and positive definite matrices $\Phi, \Theta$ and $\Psi$ such that

$$
\left[\begin{array}{cccc}
-\Phi & 0 & 0 & X_{0}^{\top} \Psi \\
0 & -\Theta & 0 & X_{1}^{\top} \Psi \\
0 & 0 & -(\Psi-\Phi-\Theta) & X_{2}^{\top} \Psi \\
\Psi X_{0} & \Psi X_{1} & \Psi X_{2} & -\Psi
\end{array}\right]<0
$$

Pre- and post-multiplying this matrix inequality by $\operatorname{diag}\left\{\Psi^{-1}, \Psi^{-1}, \Psi^{-1}, \Psi^{-1}\right\}$ and defining $M=\Psi^{-1} \boldsymbol{\Phi} \Psi^{-1}$, $N=\Psi^{-1} \Theta \Psi^{-1}, Z=\Psi^{-1}$, and $E=K \Psi^{-1}$, yields (24). Finally, note that $K=E Z^{-1}$.

When (22) holds, the matrices $H_{i}$ in (23) can be considered void. In this case, condition (24) in Theorem 2 reduces to the existence $M=M^{\top}>0, N=N^{\top}>0$ and $Z=Z^{\top}>0$ satisfying the LMI

$$
\left[\begin{array}{cccc}
-M & 0 & 0 & Z L_{0}^{\top} \\
0 & -N & 0 & Z L_{1}^{\top} \\
0 & 0 & -(Z-M-N) & Z L_{2}^{\top} \\
L_{0} Z & L_{1} Z & L_{2} Z & -Z
\end{array}\right]<0
$$

which is obviously another way of saying that $\left(L_{0}, L_{1}, L_{2}\right)$ satisfies the sufficient condition for stability (7). As mentioned above, in this case there is only one solution $(X, \Omega)$ of equation (10), so that there are no degrees of freedom in the choice of $F_{\Omega}$.

\section{A. External stabilisation for controlled invariance}

Given a controlled invariant subspace $\mathscr{V}$ and a corresponding basis matrix $V$, let $(X, \Omega)$ be any solution of $(10)$ and let $F_{\Omega}=-\Omega\left(V^{\top} V\right)^{-1} V^{\top}$ be a friend of $\mathscr{V}$ that is internally stabilising. We now consider the possibility of choosing a suitable $\tilde{F}$ in order to stabilise $\mathscr{V}$ externally. Applying the static feedback control action $u_{i, j}=\left(F_{\Omega}+\tilde{F}\right) x_{i, j}$ in (1) yields

$$
\begin{aligned}
x_{i+1, j+1} & =\left(\Xi_{0, \Omega}+B_{0} \tilde{F}\right) x_{i, j}+\left(\Xi_{1, \Omega}+B_{1} \tilde{F}\right) x_{i+1, j} \\
& =+\left(\Xi_{2, \Omega}+B_{2} \tilde{F}\right) x_{i, j+1},
\end{aligned}
$$

where $\Xi_{i, \Omega} \triangleq A_{i}+B_{i} F_{\Omega}$. The problem can now be considered as one of finding $\tilde{F}$ such that

$$
\left\{\begin{array}{l}
\left(\Xi_{0, \Omega}+B_{0} \tilde{F}, \Xi_{1, \Omega}+B_{1} \tilde{F}, \Xi_{2, \Omega}+B_{2} \tilde{F}\right) \text { asympt. stable } \\
\tilde{F} V=0
\end{array}\right.
$$

Theorem 3: Let $\mathscr{V}$, with basis matrix $V$, be a controlledinvariant subspace for (1). Suppose that $\mathscr{V}$ is internally stabilised by the static feedback matrix $F_{\Omega}$; i.e., $\left(\Xi_{0, \Omega}, \Xi_{1, \Omega}, \Xi_{2, \Omega}\right)$ is internally stable with respect to $\mathscr{V}$. Then $\mathscr{V}$ is also externally stabilisable if there exist matrices $M=M^{\top}>0, N=N^{\top}>0, Z=Z^{\top}>0, R=R^{\top}>0$ and $S$ of suitable dimensions such that

$$
\left[\begin{array}{cccc}
-M & 0 & 0 & \tilde{\Xi}_{0, \Omega}^{\top} \\
0 & -N & 0 & \tilde{\Xi}_{1, \Omega}^{\top} \\
0 & 0 & -(Z-M-N) & \tilde{\Xi}_{2, \Omega}^{\top} \\
\tilde{\Xi}_{0, \Omega} & \tilde{\Xi}_{1, \Omega} & \tilde{\Xi}_{2, \Omega} & -R
\end{array}\right]<0
$$

with

$$
Z R=I
$$

where $\tilde{\Xi}_{i, \Omega} \triangleq \Xi_{i, \Omega}+B_{i} S^{\top} \tilde{V}^{\top}$ and $\tilde{V}$ is a basis matrix for $\operatorname{ker} V^{\top}$.

Proof: First note that the condition $\tilde{F} V=0$ can also be written as $\operatorname{im} \tilde{F}^{\top} \subseteq \operatorname{ker} V^{\top}$. Then, consider a basis matrix $\tilde{V}$ of $\operatorname{ker} V^{\top}$, so that $\operatorname{im} \tilde{F}^{\top} \subseteq \operatorname{im} \tilde{V}$. As such, $\tilde{F}^{\top}=\tilde{V} S$ for some matrix $S$ so that $\tilde{F}=S^{\top} \tilde{V}^{\top}$. Now by Lemma 3 , the triple $\left(\tilde{\Xi}_{0, \Omega}, \tilde{\Xi}_{1, \Omega}, \tilde{\Xi}_{2, \Omega}\right)$ is asymptotically stable if there exist three symmetric positive definite matrices $M, N, Z$ and a matrix $S$ of suitable dimension such that

$$
\left[\begin{array}{cccc}
-M & 0 & 0 & \tilde{\Xi}_{0, \Omega}^{\top} \\
0 & -N & 0 & \tilde{\Xi}_{1, \Omega}^{\top} \\
0 & 0 & -(Z-M-N) & \tilde{\Xi}_{2, \Omega}^{\top} \\
\tilde{\Xi}_{0, \Omega} & \tilde{\Xi}_{1, \Omega} & \tilde{\Xi}_{2, \Omega} & -Z^{-1}
\end{array}\right]<0
$$

which is equivalent to (25) when combined with (26).

The set defined by the inequality (25) with the constraint (26) is not convex. However, various established numerical techniques are available for finding feasible points. Here we consider the so-called sequential linear programming matrix method (SLPMM) developed in [13]. To this end, we first notice that condition (26) is satisfied if and only if $\operatorname{Trace}(Z R)=n$ and

$$
\left[\begin{array}{ll}
Z & I \\
I & R
\end{array}\right] \geq 0
$$

The problem of finding $(M, N, Z, R, S)$ that satisfy (25-26) can then be tackled with the following algorithm. ${ }^{2}$

Algorithm 1: ([13])

Step 1: Check the existence of a pair $(Z, R)$ satisfying (25) and (27). If such pair exists, denote it with $\left(Z^{0}, R^{0}\right)$.

\footnotetext{
${ }^{2}$ This may not always yield a feasible point, even if the non-convex set defined by $(25-26)$ is non-empty.
} 
Step 2: Given $\left(Z^{k}, R^{k}\right), k \geq 0$, obtain a solution $(Z, R)$ together with $S$, to the convex optimization problem

$$
\begin{aligned}
& \min \operatorname{Trace}\left(Z R^{k}+Z^{k} R\right) \\
& \text { subject to }(25),(27) .
\end{aligned}
$$

Denote this solution with $\left(Z_{T}^{k}, R_{T}^{k}\right)$.

Step 3: If $\left|\operatorname{Trace}\left(Z_{T}^{k} R^{k}+Z^{k} R_{T}^{k}\right)-2 \cdot \operatorname{Trace}\left(Z^{k} R^{k}\right)\right| \leq v$ then stop, where $v$ is a sufficiently small positive scalar.

Step 4: Compute $\alpha \in[0,1]$ by solving

$$
\min _{\alpha \in[0,1]} \operatorname{Trace}\left(\left[Z^{k}+\alpha\left(Z_{T}^{k}-Z^{k}\right)\right]\left[R^{k}+\alpha\left(R_{T}^{k}-R^{k}\right)\right]\right) .
$$

Step 5: Set $Z^{k+1}=(1-\alpha) Z^{k}+\alpha Z_{T}^{k}$ and $R^{k+1}=(1-\alpha) R^{k}+$ $\alpha R_{T}^{k}$, then go to Step 2 .

\section{CONDITIONED INVARIANT SUBSPACES}

Now we focus our attention on the definition of conditioned invariant subspaces.

Definition 4: The subspace $\mathscr{S} \subseteq \mathbb{R}^{n}$ is conditioned invariant for (1) if $A_{H}\left(\mathscr{S}_{D} \cap \operatorname{ker} C_{D}\right) \subseteq \mathscr{S}$.

It is can be seen that the set of conditioned invariant subspaces is closed under subspace intersection but not under subspace addition. Its smallest element is $\mathbf{0}_{n}$, its largest element is $\mathbb{R}^{n}$.

Lemma 7: Let $\mathscr{S}$ be an $s$-dimensional subspace of $\mathbb{R}^{n}$, and let $Q \in \mathbb{R}^{(n-s) \times n}$ be such that $\operatorname{ker} Q=\mathscr{S}$ with $Q$ of full row-rank. The following statements are equivalent:

1) the subspace $\mathscr{S}$ is conditioned invariant for (1);

2) there exist matrices $\Gamma=\left[\Gamma_{0} \Gamma_{1} \Gamma_{2}\right] \in \mathbb{R}^{(n-s) \times 3(n-s)}$ and $\Lambda=\left[\begin{array}{lll}\Lambda_{0} & \Lambda_{1} & \Lambda_{2}\end{array}\right] \in \mathbb{R}^{(n-s) \times 3 p}$ such that

$$
Q A_{H}=\Gamma Q_{D}+\Lambda C_{D} ;
$$

3) there exists a matrix $G=\left[G_{0} G_{1} G_{2}\right] \in \mathbb{R}^{n \times 3 p}$ such that

$$
\left(A_{H}+G C_{D}\right) \mathscr{S}_{D} \subseteq \mathscr{S} \text {. }
$$

Proof: 1) $\Longrightarrow 2$ 2). Since $\mathscr{S}$ is such that $A_{H}\left(\mathscr{S}_{D} \cap \operatorname{ker} C_{D}\right) \subseteq$ $\mathscr{S}$, it follows that $\operatorname{ker}\left[\begin{array}{l}Q_{D} \\ C_{D}\end{array}\right] \subseteq \operatorname{ker} Q A_{H}$ and as such, there exist $\Gamma \in \mathbb{R}^{(n-s) \times 3(n-s)}$ and $\Lambda \in \mathbb{R}^{(n-s) \times 3 p}$ such that $Q A_{H}=$ $\Gamma Q_{D}+\Lambda C_{D}$; see Proof of Lemma 1. 2) $\Longrightarrow 3$ ). Equation (29) follows from (28) with any $G$ such that $\Lambda=-Q G$.3) $\Longrightarrow 1$ ). This follows by definition.

Property 3) in Lemma 7 means that $\mathscr{S}$ is conditioned invariant for (1) if and only if there exists an outputinjection matrix $G=\left[G_{0} G_{1} G_{2}\right] \in \mathbb{R}^{n \times 3 p}$ such that $\mathscr{S}$ is a $\left(A_{0}+G_{0} C, A_{1}+G_{1} C, A_{2}+G_{2} C\right)$-invariant subspace. Let $\Gamma$ and $\Lambda$ be such that (28) holds, which can be written as

$$
Q A_{H}=\left[\begin{array}{ll}
\Gamma & \Lambda
\end{array}\right]\left[\begin{array}{l}
Q_{D} \\
C_{D}
\end{array}\right]
$$

a linear equation which can be solve for $\Gamma$ and $\Lambda$. Given a conditioned invariant subspace, the solutions of (30) are given by

$$
\left[\begin{array}{ll}
\Gamma & \Lambda
\end{array}\right]=Q A_{H}\left[\begin{array}{l}
Q_{D} \\
C_{D}
\end{array}\right]^{\dagger}+K H
$$

where the rows of $H$ span the null-space of $\left[\begin{array}{ll}Q_{D}^{\top} & C_{D}^{\top}\end{array}\right]$ and $K$ is an arbitrary matrix of suitable size. When $\left[\begin{array}{l}Q_{D} \\ C_{D}\end{array}\right]$ is full-rank, matrix $K$ has zero rows; i.e., the only solution of (30) is $[\Gamma \Lambda]=Q A_{H}\left[\begin{array}{l}Q_{D} \\ C_{D}\end{array}\right]^{\dagger}$. By (29), $\tilde{\Gamma}$ exists such that

$$
Q\left(A_{H}+G C_{D}\right)=\tilde{\Gamma} Q_{D}
$$

We now investigate the relation between the pairs $(\Gamma, \Lambda)$ and $(G, \tilde{\Gamma})$ satisfying (30) and (32), respectively. First, notice that given a pair $(G, \tilde{\Gamma})$ such that (32) holds, then (30) is satisfied with $\Gamma=\tilde{\Gamma}$ and $\Lambda=-Q G$. Conversely, given a pair of matrices $(\Gamma, \Lambda)$ such that (30) holds, then (32) is satisfied with $\tilde{\Gamma}=\Gamma$ and with any $G$ such that $\Lambda=-Q G$. As such, no generality is lost by assuming $\tilde{\Gamma}=\Gamma$, and by representing the set of all friends of the conditioned invariant subspace $\mathscr{S}$ as the set of matrices $G \in \mathbb{R}^{n \times 3 p}$ satisfying $\Lambda=-Q G$, where $\Lambda \in \mathbb{R}^{(n-s) \times 3 p}$ is any matrix for which another matrix $\Gamma \in \mathbb{R}^{(n-s) \times 3(n-s)}$ exists so that (30) holds. For any pair $(\Gamma, \Lambda)$ such that (30) holds, the solutions of the linear equation $\Lambda=-Q G$ are parameterised as

$$
G=G_{\Lambda}+\tilde{G}
$$

where $G_{\Lambda} \triangleq-Q^{\top}\left(Q Q^{\top}\right)^{-1} \Lambda$ and $\tilde{G}$ is any $n \times 3 p$ matrix such that $Q \tilde{G}=0$, or, equivalently, such that $\operatorname{im} \tilde{G} \subseteq \operatorname{ker} Q$. The choice of $\tilde{G}$ affects the external stability of $\mathscr{S}$, but not the internal stability of $\mathscr{S}$. Similarly, $G_{\Lambda}$ can affect the internal but not the external stability of $\mathscr{S}$. With reference to the proof of Corollary 1 , note that with $S \triangleq\left[\begin{array}{l}S_{c} \\ Q\end{array}\right]$, where the rows of $S_{c}$ are linearly independent from those of $Q$, so that $Q S^{-1}=[0 I]$, we have that for all $i \in\{0,1,2\}$

$$
S\left(A_{i}+G_{i} C\right) S^{-1}=\left[\begin{array}{cc}
\Delta_{i}^{11}(\Lambda, \tilde{G}) & \Delta_{i}^{12}(\Lambda, \tilde{G}) \\
0 & \Delta_{i}^{22}(\Lambda, \tilde{G})
\end{array}\right] .
$$

Lemma 8: For all $i \in\{0,1,2\}$, the matrix $\Delta_{i}^{22}(\Lambda, \tilde{G})$ does not depend on $\tilde{G}$, and the matrix $\Delta_{i}^{11}(\Lambda, \tilde{G})$ does not depend on the particular $\Lambda$ which satisfies (30) for some $\Gamma$.

Proof: The proof follows the same line as the proof of Lemma 6.

Conditioned invariance is linked to the existence of 2-D quotient observers [15]. For an observer of the form ${ }^{3}$

$$
\begin{aligned}
\omega_{i+1, j+1}= & K_{0} \omega_{i, j}+K_{1} \omega_{i+1, j}+K_{2} \omega_{i, j+1} \\
& +L_{0} y_{i, j}+L_{1} y_{i+1, j}+L_{2} y_{i, j+1},
\end{aligned}
$$

for (1) with $u_{i, j}=0$, it follows that with $e_{i, j}:=Q x_{i, j}-\omega_{i, j}$,

$$
e_{i+1, j+1}=\left(Q A_{H}-L_{H} C_{D}\right)\left[\begin{array}{c}
x_{i, j} \\
x_{i+1, j} \\
x_{i, j+1}
\end{array}\right]+K_{H}\left[\begin{array}{c}
\omega_{i, j} \\
\omega_{i+1, j} \\
\omega_{i, j+1}
\end{array}\right] .
$$

For $K_{H}=\Gamma$ and $L_{H}=\Lambda$, where $(\Lambda, \Gamma)$ satisfy (30), this becomes

$$
\begin{aligned}
e_{i+1, j+1} & =\Gamma Q_{D}\left[\begin{array}{c}
x_{i, j} \\
x_{i+1, j} \\
x_{i, j+1}
\end{array}\right]+\Gamma\left[\begin{array}{c}
\omega_{i, j} \\
\omega_{i+1, j} \\
\omega_{i, j+1}
\end{array}\right] \\
& =\Gamma_{0} e_{i, j}+\Gamma_{1} e_{i+1, j}+\Gamma_{2} e_{i, j+1},
\end{aligned}
$$

so that with observer boundary conditions $\omega_{i, j}=x_{i, j} / \mathscr{S}$, for $(i, j) \in \mathfrak{B}$, it follows that $e_{i, j}=0$ for $(i, j) \in \mathfrak{B}$, and hence,

\footnotetext{
${ }^{3}$ This is different to the form considered in [15]
} 
all $(i, j) \in \mathbb{N} \times \mathbb{N}$ by (37). If $\left(\Gamma_{0}, \Gamma_{1}, \Gamma_{2}\right)$ is asymptotically stable, then the observer is said to be asymptotic in the sense that $e_{i, j} \rightarrow 0$ as $i+j \rightarrow 0$ for any boundary conditions. In view of Corollary 1, part 2), we are therefore interested in finding $G=\left[G_{0} G_{1} G_{2}\right]$ such that $\mathscr{S}$ is an externally stable $\left(A_{0}+G_{0} C, A_{1}+G_{1} C, A_{2}+G_{2} C\right)$-invariant subspace; i.e., such that there exists an asymptotically stable triple $\left(\Gamma_{0}, \Gamma_{1}, \Gamma_{2}\right)$ for which $Q\left(A_{H}+G C_{D}\right)=\Gamma Q_{D}$. When such a $G$ exists, $\mathscr{S}$ is called a detectability subspace.

For a given a conditioned invariant $\mathscr{S}$, write (31) as

$\left[\begin{array}{llll}\Gamma_{0} & \Gamma_{1} & \Gamma_{2} & \Lambda\end{array}\right]=\left[\begin{array}{llll}V_{0} & V_{1} & V_{2} & V_{3}\end{array}\right]+K\left[\begin{array}{llll}H_{0} & H_{1} & H_{2} & H_{3}\end{array}\right]$,

where $\left[\begin{array}{llll}V_{0} & V_{1} & V_{2} & V_{3}\end{array}\right]=Q A_{H}\left[\begin{array}{l}Q_{D} \\ C_{D}\end{array}\right]^{\dagger}$ and the rows of $\left[\begin{array}{llll}H_{0} & H_{1} & H_{2} & H_{3}\end{array}\right]$, partitioned comformably with $\left[\begin{array}{llll}\Gamma_{0} & \Gamma_{1} & \Gamma_{2} & \Lambda\end{array}\right]$, span the kernel of $\left[\begin{array}{ll}Q_{D}^{\top} & C_{D}^{\top}\end{array}\right]$. If this null space is zero, i.e., if $\mathscr{S}_{D}+\operatorname{ker} C_{D}=\mathbb{R}^{3 n}$, there is only one solution to (30), so that there are no degrees of freedom in the choice of the triple $\left(\Gamma_{0}, \Gamma_{1}, \Gamma_{2}\right)$. In this case, if $\left(\Gamma_{0}, \Gamma_{1}, \Gamma_{2}\right)=\left(V_{0}, V_{1}, V_{2}\right)$ is stable, then with the corresponding $\Lambda=\left[\Lambda_{0} \Lambda_{1} \Lambda_{2}\right]=V_{3}$, the matrix $G_{\Lambda} \triangleq-Q^{\top}\left(Q Q^{\top}\right)^{-1} \Lambda=\left[G_{\Lambda, 0} G_{\Lambda, 1} G_{\Lambda, 2}\right]$ is such that $\mathscr{S}$ is an externally stable $\left(A_{0}+G_{\Lambda, 0} C, A_{1}+G_{\Lambda, 1} C, A_{2}+G_{\Lambda, 2} C\right)$ invariant subspace. On the other hand, if the triple $\left(\Gamma_{0}, \Gamma_{1}, \Gamma_{2}\right)=\left(V_{0}, V_{1}, V_{2}\right)$ is not asymptotically stable, the subspace $\mathscr{S}$ is not a detectability subspace.

Now, when $\mathscr{S}_{D}+\operatorname{ker} C_{D} \subset \mathbb{R}^{3 n}$, the problem we need to solve is to find a matrix $K$ such that the resulting triple $\left(\Gamma_{0}, \Gamma_{1}, \Gamma_{2}\right)=\left(V_{0}+K H_{0}, V_{1}+K H_{1}, V_{2}+K H_{2}\right)$ is asymptotically stable; the corresponding $\Lambda=\left[\Lambda_{0} \Lambda_{1} \Lambda_{2}\right]=V_{3}+K H_{3}$, for which $(\Gamma, \Lambda)$ is a solution of (28), is such that $G_{\Lambda} \triangleq$ $-Q^{\top}\left(Q Q^{\top}\right)^{-1} \Lambda$, yielding $Q\left(A_{H}+G_{\Lambda} C_{D}\right)=\Gamma Q_{D}$, so that $\mathscr{S}$ is an externally stable $\left(A_{0}+G_{\Lambda, 0} C, A_{1}+G_{\Lambda, 1} C, A_{2}+G_{\Lambda, 2} C\right)$ invariant subspace. Towards characterising a subset of such matrices $K$, we can rewrite the sufficient condition for asymptotic stability in Lemma 3 for the triple $\left(\Gamma_{0}, \Gamma_{1}, \Gamma_{2}\right)$ as shown below

$$
\left[\begin{array}{ccc}
\Phi & 0 & 0 \\
0 & \Psi & 0 \\
0 & 0 & \Theta-\Phi-\Psi
\end{array}\right]-\left[\begin{array}{c}
\Gamma_{0}^{\top} \\
\Gamma_{1}^{\top} \\
\Gamma_{2}^{\top}
\end{array}\right] \Theta\left[\begin{array}{ccc}
\Gamma_{0} & \Gamma_{1} & \Gamma_{2}
\end{array}\right]>0,
$$

for some $\Phi \triangleq P_{0}>0, \Psi \triangleq P_{1}>0$ and $\Theta \triangleq P_{0}+P_{1}+P_{2}>0$. Standard manipulation and $\Gamma_{i}=V_{i}+K H_{i}$, for $i=0,1,2$, yield the equivalent condition

$$
\left[\begin{array}{cccc}
\Phi & 0 & 0 & \tilde{V}_{i}^{\top} \\
0 & \Psi & 0 & \tilde{V}_{1}^{\top} \\
0 & 0 & \Theta-\Phi-\Psi & \tilde{V}_{2}^{\top} \\
\tilde{V}_{0} & \tilde{V}_{1} & \tilde{V}_{2} & \Theta
\end{array}\right]>0
$$

for some $\Phi>0, \Psi>0, \Theta>0$ and $\Pi$ of suitable dimensions, where $\tilde{V}_{i} \triangleq \Theta V_{i}+\Pi H_{i}$ and $\Pi=\Theta K$.

Theorem 4: Let $\mathscr{S}$ be a conditioned invariant subspace for (1), $\left[\begin{array}{llll}V_{0} & V_{1} & V_{2} & V_{3}\end{array}\right]=Q A_{H}\left[\begin{array}{l}Q_{D} \\ C_{D}\end{array}\right]^{\dagger}$ and $\left[\begin{array}{llll}H_{0} & H_{1} & H_{2} & H_{3}\end{array}\right]$ be such that its rows are a basis for the kernel of $\left[\begin{array}{ll}Q_{D}^{\top} & C_{D}^{\top}\end{array}\right]$. The subspace $\mathscr{S}$ is a detectability subspace if there exist $\Phi=\Phi^{\top}>0, \Psi=\Psi^{\top}>0, \Theta=\Theta^{\top}>0$ and $\Pi$ of suitable dimensions such that (38) holds. Moreover, given a quadruple $(\Theta, \Phi, \Psi, \Pi)$ in the convex set defined by (38), a matrix $K$ for which the triple $\left(\Gamma_{0}, \Gamma_{1}, \Gamma_{2}\right)$ is asymptotically stable is given by $K=\Theta^{-1} \Pi^{\top}$.

\section{OUTPUT-NULLING AND INPUT-CONTAINING SUBSPACES}

We now turn our attention to output-nulling subspaces, which play a fundamental role in several control problems, like disturbance localisation and decoupling, non-interaction and optimal control problems. These are a particular type of controlled invariant subspaces for (1). The subspace $\mathscr{V} \subseteq \mathbb{R}^{n}$ is an output-nulling subspace for (1) if

$$
\left[\begin{array}{c}
A_{V} \\
C
\end{array}\right] \mathscr{V} \subseteq\left(\mathscr{V}_{D} \times \mathbf{0}_{p}\right)+\operatorname{im}\left[\begin{array}{c}
B_{V} \\
D
\end{array}\right]
$$

An output-nulling subspace $\mathscr{V}$ is such that for any $\mathscr{V}$-valued boundary condition, there is an input function for which the local state trajectory of (1) lies in $\mathscr{V}$ and the corresponding output is zero for all $(i, j)$ such that $i, j \geq 0$. Such an input can always be expressed as a static state feedback.

Lemma 9: Let $V$ be a basis matrix for an $r$-dimensional subspace $\mathscr{V} \subseteq \mathbb{R}^{n}$. The following statements are equivalent:

1) The subspace $\mathscr{V}$ is output-nulling for (1).

2) Two matrices $X \in \mathbb{R}^{3 r \times r}$ and $\Omega \in \mathbb{R}^{m \times r}$ exist such that

$$
\left[\begin{array}{c}
A_{V} \\
C
\end{array}\right] V=\left[\begin{array}{c}
V_{D} \\
0
\end{array}\right] X+\left[\begin{array}{c}
B_{V} \\
D
\end{array}\right] \Omega
$$

3) A matrix $F \in \mathbb{R}^{m \times n}$ exists such that

$$
\left[\begin{array}{c}
A_{V}^{F} \\
C+D F
\end{array}\right] \mathscr{V} \subseteq \mathscr{V}_{D} \times \mathbf{0}_{p}
$$

The set of output-nulling controlled invariant subspaces is denoted with the symbol $\mathfrak{V}_{0}$. Given a $\mathscr{V} \in \mathfrak{V}_{0}$, any matrix $F$ such that (41) holds is called an output-nulling friend. It is not difficult to see that, as in the 1-D case, the set $\mathfrak{V}_{0}$ is closed under subspace addition. Thus, the sum of all output-nulling subspaces for (1) is the largest output-nulling subspace and this is denoted by $\mathscr{V}^{\star}$. The following enables computation of $\mathscr{V}^{\star}$ in finite terms, as the $(n-1)$-th term of a monotonically non-increasing sequence of subspaces.

Algorithm 2: The sequence of subspaces $\left(\mathscr{V}^{i}\right)_{i \in \mathbb{N}}$ described by the recurrence

$$
\begin{aligned}
& \mathscr{V}^{0}=\mathbb{R}^{n} \\
& \mathscr{V}^{i}=\left[\begin{array}{c}
A_{V} \\
C
\end{array}\right]^{-1}\left(\left(\mathscr{V}_{D}^{i-1} \times \mathbf{0}_{p}\right)+\operatorname{im}\left[\begin{array}{c}
B_{V} \\
D
\end{array}\right]\right), i>0,
\end{aligned}
$$

is monotonically non-increasing. Moreover, there exists an integer $k \leq n-1$ such that $\mathscr{V}^{k+1}=\mathscr{V}^{k}$. For such $k$ the identity $\mathscr{V}^{\star}=\mathscr{V}^{k}$ holds.

Algorithm 2 is a generalisation of a corresponding result in [4, Proposition 2.7], to the case of 'non-strictly proper' systems. Due to the invariance property (41) of the set of all output-nulling friends associated with the elements of the output-nulling controlled invariant subspaces $\mathfrak{V}_{0}$ for (1), we can introduce the notions of internal stabilisability and external stabilisability for output-nulling subspaces: An 
output-nulling subspace $\mathscr{V} \in \mathfrak{V}_{0}$ is said to be internally stabilisable (resp. externally stabilisable) if there exists an output-nulling friend $F$ such that $\mathscr{V}$ is an internally stable (resp. externally stable) $\left(A_{0}^{F}, A_{1}^{F}, A_{2}^{F}\right)$-invariant.

Given a $\mathscr{V}$-valued boundary condition for (1) with $\mathscr{V} \in$ $\mathfrak{V}_{0}$, any control action $u_{i, j}=F x_{i, j}$ with $F$ satisfying (41) is such that $x_{i, j} \in \mathscr{V}$ and $y_{i, j}=0$ for all $i, j$ such that $i, j \geq 0$. To see this, it suffices to substitute $u_{i, j}=F x_{i, j}$ in (1) to get

$$
\begin{aligned}
x_{i+1, j+1} & =A_{0}^{F} x_{i, j}+A_{1}^{F} x_{i+1, j}+A_{2}^{F} x_{i, j+1} \\
y_{i, j} & =(C+D F) x_{i, j}
\end{aligned}
$$

and to observe that when $x_{i, j}, x_{i+1, j}$ and $x_{i, j+1}$ belong to $\mathscr{V}$, so does $x_{i+1, j+1}$ because of (41). As a result, for any $\mathscr{V}$-valued boundary condition it is found that $x_{i, j} \in \mathscr{V}$ and $y_{i, j}=0$ since $\mathscr{V} \subseteq \operatorname{ker}(C+D F)$. This shows that the control input required to maintain the output at zero and the local state on $\mathscr{V}$ can always be expressed as a static local state feeback. As such, all of the material developed in Section III for controlled invariant subspaces can be adapted straightforwardly to output-nulling subspaces with few modifications. Indeed, by replacing (10) with (40) and (22) with $\mathscr{V}_{D} \cap B_{V} \operatorname{ker} D=\mathbf{0}_{3 n}$, the internal and external stabilisation of output-nulling subspaces via output-nulling static feedback can be carried out along the same lines as the internal and external stabilisation of arbitrary controlled invariant subspaces.

Now we turn our attention to input-containing subspaces, which are particular types of conditioned-invariant subspaces useful in the context of various filtering/estimation problems, like unknown-input observation and fault detection.

Definition 5: We define a input-containing subspace $\mathscr{S}$ for (1) as a subspace of $\mathbb{R}^{n}$ such that

$$
\left[\begin{array}{ll}
A_{H} & B_{H}
\end{array}\right]\left(\left(\mathscr{S}_{D} \times \mathbb{R}^{3 m}\right) \cap \operatorname{ker}\left[\begin{array}{ll}
C_{D} & D_{D}
\end{array}\right]\right) \subseteq \mathscr{S} .
$$

The set of input-containing subspaces for (1) is denoted by the symbol $\mathfrak{S}_{0}$. The intersection of two input-containing subspaces is input-containing. It follows that the set $\mathfrak{S}_{0}$ is closed under subspace intersection. The same is not true for subspace addition. The intersection of all the inputcontaining subspaces of $\Sigma$ is the smallest input-containing subspace of $\Sigma$, and is usually denoted by $\mathscr{S}^{\star}$.

Lemma 10: Given the $s$-dimensional subspace $\mathscr{S}$ of $\mathbb{R}^{n}$, let $Q \in \mathbb{R}^{(n-s) \times n}$ be such that $\operatorname{ker} Q=\mathscr{S}$ with $Q$ of full rowrank. The following statements are equivalent:

1) the subspace $\mathscr{S}$ is input-containing for (1);

2) two matrices $\Gamma \in \mathbb{R}^{(n-s) \times 3(n-s)}$ and $\Lambda \in \mathbb{R}^{(n-s) \times 3 p}$ exist such that

$$
Q\left[\begin{array}{ll}
A_{H} & B_{H}
\end{array}\right]=\Gamma\left[\begin{array}{ll}
Q_{D} & 0
\end{array}\right]+\Lambda\left[\begin{array}{ll}
C_{D} & D_{D}
\end{array}\right]
$$

3) a matrix $G \in \mathbb{R}^{n \times 3 p}$ exists such that

$$
\left[A_{H}+G C_{D} \quad B_{H}+G D_{D}\right]\left(\mathscr{S}_{D} \times \mathbb{R}^{3 m}\right) \subseteq \mathscr{S}
$$

The following is an algorithm for computing the smallest input-containing subspace $\mathscr{S}^{\star}$.
Algorithm 3: The sequence of subspaces $\left(\mathscr{S}^{i}\right)_{i \in \mathbb{N}}$ described by the recurrence

$$
\begin{aligned}
\mathscr{S}^{0} & =0_{n} \\
\mathscr{S}^{i} & =\left[\begin{array}{ll}
A_{H} & B_{H}
\end{array}\right]\left(\left(\mathscr{S}_{D}^{i-1} \times \mathbb{R}^{3 m}\right) \cap \operatorname{ker}\left[\begin{array}{ll}
C_{D} & D_{D}
\end{array}\right]\right),
\end{aligned}
$$

for $i>0$, is monotonically non-increasing. An integer $k \leq n-1$ exists such that $\mathscr{S}^{k+1}=\mathscr{S}^{k}$. For such $k$, the identity $\mathscr{S}^{\star}=\mathscr{S}^{k}$ holds.

\section{CONCLUSIONS}

The problem of internal and external stabilisation of controlled and conditioned invariant subspaces has been considered for 2-D Fornasini-Marchesini models in the general form of Kurek. The main results enable the extension of various geometric analysis and synthesis results for 2-D systems already available in the literature by accommodating stability requirements. These include geometric approaches to problems of disturbance decoupling with unaccessible, measureable and previewed signals, model matching problems, full information problems, non-interaction control problems, unknown-input observation and fault detection problems.

\section{REFERENCES}

[1] G. Basile and G. Marro. Controlled and conditioned invariant subspaces in linear system theory. J. Opt. Theory Appl., 3(5):306$315,1969$.

[2] M. Bisiacco and M.E. Valcher. Unknown input observers for 2D statespace models. International Journal of Control, vol. 77, no. 9, pp. 861876(16), June 10, 2004

[3] P.A. Bliman, Lyapunov Equation for the Stability of 2-D Systems. Multidimensional Systems and Signal Processing, 13:201-222, 2002.

[4] G. Conte and A. Perdon. A geometric approach to the theory of 2-D systems. IEEE Trans. Aut. Control, AC-33(10):946-950, 1988.

[5] Y. Ebihara, and Y. Ito, and T. Hagiwara, Exact Stability Analysis of 2-D Systems Using LMIs. IEEE Trans. Aut. Control, AC-51(9):1509$1513,2006$.

[6] E. Fornasini and G. Marchesini. Doubly-Indexed Dynamical Systems: State-Space Models and Structural Properties. Mathematical System Theory, 12:59-72, 1978.

[7] E. Fornasini and G. Marchesini, Stability analysis of 2-D systems. IEEE Transactions on Circuits and Systems, CAS-27(12):1210-1217, 1980.

[8] K. Gałkowski, The Fornasini-Marchesini and the Roesser models: algebraic methods for recasting, IEEE Transactions on Automatic Control, vol. 41, pp. 107-112, 1996.

[9] G.D. Hu and M. Liu. Simple Criteria for Stability of Two-Dimensional Linear Systems. IEEE Trans. Sign. Process., 53(12):4720-4723, 2005.

[10] H. Kar and V. Sigh. Stability of 2-D systems described by the Fornasini-Marchesini first model. IEEE Trans. Sign. Process., 51(6):1675-1676, 2003.

[11] A. Karamanciog̃lu and F.L. Lewis. Geometric theory for the singular Roesser model. IEEE Trans. Aut. Control, AC-37(6):801-806, 1992.

[12] J. E. Kurek, The general state-space model for a two-dimensional linear digital systems, IEEE Transactions on Automatic Control, vol. 30, no. 6, pp. 600-602, 1985.

[13] F. Leibfritz, An LMI-based algorithm for designing suboptimal static $\mathscr{H}_{2} / \mathscr{H}_{\infty}$ output feedback controllers. SIAM J. Contr. Optim., 39(6):1711-1735, 2001.

[14] L. Ntogramatzidis, M. Cantoni, and R. Yang, A geometric theory for 2-D systems including notions of stabilisability. Multidimensional Systems and Signal Processing, 19: 449-475, 2008.

[15] L. Ntogramatzidis and M. Cantoni, Conditioned invariance and unknown-input observation for two-dimensional Fornasini-Marchesini models. In Proceedings of European Control Conference, Kos, 2007.

[16] R.P. Roesser, A discrete state-space model for linear image processing. IEEE Trans. Aut. Control, AC-20(1):1-10, 1975.

[17] T. Zhou, Stability and Stability Margin for a Two-Dimensional System. IEEE Transactions on Signal Processing, 54(9):3483-3488, September 2006. 